AperTO - Archivio Istituzionale Open Access dell'Università di Torino

\title{
CEST-MRI studies of cells loaded with lanthanide shift reagents
}

\section{This is a pre print version of the following article:}

Original Citation:

Availability:

This version is available http://hdl.handle.net/2318/1691701

since 2019-02-11T13:56:37Z

Published version:

DOI: $10.1002 / \mathrm{mrm} .27157$

Terms of use:

Open Access

Anyone can freely access the full text of works made available as "Open Access". Works made available under a Creative Commons license can be used according to the terms and conditions of said license. Use of all other works requires consent of the right holder (author or publisher) if not exempted from copyright protection by the applicable law. 


\title{
CEST-MRI studies of cells loaded with Lanthanide Shift reagents
}

Ferrauto Giuseppe ${ }^{1 *}$, Di Gregorio Enza ${ }^{1}$, Daniela Delli Castelli ${ }^{1}$, Aime Silvio ${ }^{1}$

\author{
${ }^{1}$ Molecular Imaging Center, Department of Molecular Biotechnologies and Health Sciences, \\ University of Torino (IT) \\ ${ }^{*}$ Dr. Giuseppe Ferrauto \\ Molecular Imaging Center, Department of Molecular Biotechnologies and Health Sciences, \\ University of Torino, via Nizza 52, 10126, Torino, It \\ Phone: +390116708459 \\ Mail: giuseppe.ferrauto@unito.it;
}

\section{Running title: Cells as CEST agents}

Word count: 5110

Key words: MRI, CEST, Cell-CEST, Lanthanide, cell labeling, shift reagents 


\section{Abstract}

Purpose: Magnetic resonance Imaging (MRI) has been extensively used to track in vivo implanted cells that have been previously labelled with relaxation enhancers. However, this approach is not suitable to track multiple cell populations as well as it may lead to confunding results in the case the contrast agent is released from the labelled cells. Herein, it is shown that the use of Chemical Exchange Saturation Transfer (CEST) agents allows to overcome these issues. Upon encapsulating paramagnetic Lanthanide Shift Reagents (SRs) one may shift the absorption frequency of the intracellular water resonance $\left(\delta^{\ln }\right)$ thus generating frequency-encoding CEST responsive cells that can be visualized in the MR image by applying the proper radiofrequency irradiation.

Methods: Eu, Dy and Tm-HPDO3A have been used as Shift Reagents for labeling murine breast cancer cells (TS/A) and murine macrophages (J774A.1) by hypotonic swelling and pinocytosis. CEST-MR images have been acquired at 7T and Saturation Transfer effect has been measured. Samples at different dilution of cells have been analyzed to quantify the detection thresold. In vitro experiments of cells proliferation have been carried out. Finally, TS/A cells have been subcutaneously injected in mice and MR images have been acquired to assess the proliferation index in vivo.

Results: It has been found that entrapment of the paramagnetic complexes into endosomes (i.e. by the pinocytosis route) leads to an enhanced shift of the intracellular water resonance. $\delta^{\ln }$ appears to be proportional to the effective magnetic moment ( $\left.\mu^{\text {eff }}\right)$ and to the concentration of the loaded Lanthanide complex.

Moreover, a higher shift is present when the complexes are entrapped in the endosomes.

Cells proliferation index has been assessed both in vitro and in vivo by evaluating the reduction of $\delta^{\text {In }}$ value in the days after the cells labeling

\section{Conclusions:}

Cells can be visualized by CEST-MRI upon loading with paramagnetic shift reagent, by exploiting the large ensemble of the properly shifted intracellular water molecules. A better performance is obtained when the complexes are entrapped inside the endosomes. The observed $\left(\delta^{\ln }\right)$ value is strongly correlated to the chemical nature of the probe and to its concentration and cellular localization. Two applications of this method have been herein reported, i.e. i) for in vivo cells visualization and ii) for the monitoring of the cellular proliferation process as it is accompanied by a change in $\delta^{\ln }$ that, therefore, may be exploited as longitudinal reporter of the proliferation rate. 


\section{Introduction}

Cell therapy is emerging as a novel branch in many medical fields for its high potential to cure different diseases, allowing repairing of damaged or destroyed tissues ${ }^{1-2}$. Much attention is currently devoted to develop Imaging strategies able to report about the fate of cells after their in vivo transplant ${ }^{3-5}$. Among the existing Imaging technique, Magnetic Resonance (MRI) stands out owing to its excellent spatial resolution and for its ability to reach in depth regions of the body. To be detected, the administered cells have to be labelled with a proper contrast agent ${ }^{6-8}$. Conventional MRI contrast agents (CAs), based on Gd complexes or iron oxide nano-particles, have proven to be well suitable for in vivo cell detection although they have the limitation to be able to visualize only a single cell population in a given anatomical region ${ }^{9-11}$. This limitation is intrinsic in the nature of relaxation-based MRI contrast agents as their effect on water proton relaxation rate is additive thus it is not possible distinguishing the contrast generated by the one or the other CA simultaneously present in the same image.

Moreover, another issue may arise in the case the agent is released by the labelled cells as its effects would not be distinguishable when arising from its intracellular or extracellular distribution.

One of the main aims in the tracking of labeled cells is to visualize simultaneously two, or more, cellular populations and, eventually, to assess their proliferation rate.

A unique approach to acquire "multicolor" MR images is provided by the exploitation of the frequency-encoded contrast of Chemical Exchange Saturation Transfer (CEST) agents ${ }^{12-14}$. This recently developed class of MRI probes consists of molecules endowed with mobile protons in slow exchange with bulk water. The application of a radiofrequency field, at the NMR absorption frequency of the mobile proton pool, yields saturation of the NMR resonance that, through the chemical exchange, is transferred to the bulk water protons signal. It follows that the availability of systems endowed with mobile protons resonating at different chemical shifts can be distinguished when present in the same anatomical region ${ }^{15,16}$.

We have previously reported a proof of concept on the possibility to distinguish among different cells populations, in a MR-CEST experiment, by labeling each cell type with a different paraCEST (paramagnetic CEST) agent ${ }^{16}$. Furthermore, interesting applications of this approach have been reported in the detection of heart failure ${ }^{17}$ and stroke injuries ${ }^{18}$. A further improvement of this 
approach deals with the search of enhanced sensitivity of the CEST reporters as the detection threshold of paraCEST agents is in the millimolar range.

An important step ahead to enhance the sensitivity threshold has been achieved by showing that the ensembles of water molecules confined in liposomes or in the cytosol compartment may be exploited as source of exchanging proton pool ${ }^{19-20}$. In both systems the compartimentalized water resonance $\left(\delta^{\ln }\right)$ can be shifted from the absorption of the bulk solvent by the presence of a paramagnetic Shift Reagent (SR) in the inner compartment. Red Blood Cells (RBCs) have been proven to act as excellent Cell-CEST systems thanks also to their peculiar biconcave shape that greatly affect $\delta^{\ln }$ by markedly affecting the Bulk Magnetic Suceptibility (BMS) term ${ }^{20,21}$. Herein, we report results that demonstrate that other cell types, besides RBCs, can be transformed in Cell-CEST systems.

Murine breast cancer cells (TS/A) and murine macrophages (J774A.1) have been selected i) to assess which is the role of localization of the SR on the Cell-CEST properties and ii) to investigate how Cell-CEST properties may be exploited to report on the cell proliferation rate.

\section{$\underline{\text { Methods }}$}

\section{Chemicals}

Gd-HPDO3A (Gadoteridol, ProHance ${ }^{\circledR}$ ) complex and HPDO3A ligand were kindly provided by Bracco Imaging S.p.A. (Colleretto Giacosa (TO), Italy). $\mathrm{Dy}_{2} \mathrm{O}_{3}, \mathrm{Tm}_{2} \mathrm{O}_{3}$ and $\mathrm{Eu}_{2} \mathrm{O}_{3}$ were purchased from Sigma-Aldrich, Saint Louis, USA. The synthesis of Dy(III)- and Tm(III)- complexes was carried out by mixing the lanthanide oxide $\mathrm{Ln}_{2} \mathrm{O}_{3}$ and the ligand HPDO3A (1:2 molar ratio) in water. The mixture has been let to react for two weeks under stirring and heating at $80^{\circ} \mathrm{C}$. The purity of the compounds was around $90 \%$ as evaluated by measuring the bulk magnetic susceptibility (BMS) shifts of ${ }^{1} \mathrm{H}-\mathrm{NMR}$ resonance signals of solution (tert-butanol in water) containing the compound with the respect to the same solution without paramagnetic compound (Evans' Method ${ }^{22}$ ). These lanthanide complexes were dissolved in water at the concentration of $0.3 \mathrm{M}$ at a pH value ranging between 7.2-7.4. The final osmolarity of the solutions was measured by using a manual Löser type 6 Micro-Osmometer and they were about $300 \mathrm{mOsm} / \mathrm{L}$. Finally the solutions were filtered by using $0.22 \mu \mathrm{m}$ filters.

\section{$\underline{\text { Cells }}$}


Cellular experiments were performed on J774A.1 murine macrophages (ATCC, Manassass, VA) and TS/A murine breast cancer cells. J774A.1 cells were grown in Dulbecco's Modified Eagles's medium (DMEM), TS/A cells were grown on RPMI 1640 medium. Both the media were supplemented with $10 \%(\mathrm{v} / \mathrm{v})$ heat-inactivated fetal bovine serum (FBS), $100 \mathrm{U} / \mathrm{ml}$ penicillin and $100 \mathrm{mg} / \mathrm{ml}$ streptomycin.

DMEM, RPMI 1640, FBS, penicillin-streptomycin mixture and Trypsin were purchased from Lonza, (Lonza Sales AG, Verviers, Belgium). Cells were seeded in $75-\mathrm{cm}^{2}$ flasks at density of ca. $2 \times 10^{4}$ cells $/ \mathrm{cm}^{2}$ in a humidified $5 \% \mathrm{CO}_{2}$ incubator at $37{ }^{\circ} \mathrm{C}$. When $\mathrm{J774}$.A1 cells reached confluence, they were detached by means of a scraper. Conversely, TSA cells were detached by adding $1 \mathrm{ml}$ of Trypsin-EDTA solution ( $0.25 \%(\mathrm{w} / \mathrm{v})$ Trypsin- $0.53 \mathrm{mM}$ EDTA). Both the used cells types were negative for mycoplasma as tested by using MycoAlert ${ }^{\mathrm{TM}}$ Mycoplasma Detection Kit by Lonza (Lonza Sales AG, Verviers, Belgium).

\section{Cellular uptake of Shift Reagents}

SRs uptake were performed on J774.1 or TS/A cells. It was possible to introduce SRs into the endosomes or directly into the cell cytoplasm depending of the used labeling procedure. In order to introduce the SRs into the endosomes a classical procedure of incubation with Lanthanide complexes dissolved in the culture medium was performed ${ }^{23}$. Conversely, the hypotonic swelling procedure ${ }^{24}$ has been used to load the SRs into cell cytoplasm. The experimental work-up has been carried out as follows:

\section{Loading of SRs into the endosomes}

Ca $5 \times 10^{5}$ J774A.1 cells were seeded on 6-cm Petri Dishes. After 2 days plates were incubated with SRs (Ln-HPDO3A where $\mathrm{Ln}=\mathrm{Gd}$, Eu, Dy or $\mathrm{Tm}$ ) at the concentration of $100 \mathrm{mM}$ for $5 \mathrm{~h}$, then cells were washed three times, detached by scraper, suspended into $50 \mu$ of PBS and put into glass capillaries. These capillaries were centrifuged at $1000 \mathrm{rpm}$ in order to obtain cellular pellets and placed in an agar phantom to acquire MR images. MRI was carried out immediately after the detachment of cells and it requires ca. 15-20 min. During this time cells appear intact, with wellpreserved membranes. 
Time of incubation and concentration of the SRs were selected on the basis of a series of experiments. The selected conditions led to reach an intracellular concentration of SR of $c a$. $10 \mathrm{mM}$.

\section{Loading of SRs into the cytoplasm by application of hypotonic swelling methodology}

Cells were loaded by hypotonic swelling as previously reported ${ }^{24}$. Briefly, ca $3 \times 10^{6}$ cells (J774A.1 or TS/A, depending on the experiment) were placed for $30 \mathrm{~min}$ at $37^{\circ} \mathrm{C}$ into a hypotonic solution $(160 \mathrm{mOsm} / \mathrm{l})$ containing the paramagnetic agent to be loaded $(100 \mathrm{mM})$. The normal morphology of cells was restored by returning the osmolarity of the solution to an isotonic condition (280mOsm/l) by the addition of a proper concentration of $\mathrm{NaCl} /$ phosphate buffer (30min). After this treatment the samples were extensively washed by using PBS to eliminate the not internalized molecules.

\section{Determination of intracellular Lanthanide content}

At the end of MRI experiments, labeled cells were quantitatively extracted from glass capillaries, re-suspended into $200 \mu \mathrm{L}$ of PBS and sonicated by using the Bandelin Sonoplus $20 \mathrm{KHz}, 70 \mathrm{Watt}$ (Bandelin Electronic) for $20 \mathrm{sec}$ at $30 \%$ of the maximum power in order to destroy cellular membranes and obtain cell lysates. Cells were then digested with concentrated $\mathrm{HNO}_{3}(70 \%)$ under microwawe heating (Milestone MicroSYNTH Microwave labstation equipped with an optical fiber temperature control and HPR-1000/6M six position high-pressure reactor, Bergamo, Italy). Then each sample was added with $2 \mathrm{ml}$ of ultrapure water and the metal content of cells was determined using inductively coupled plasma mass spectrometry (ICP-MS) (Element-2; ThermoFinnigan, Rodano (MI), Italy). The concentration of protein was determined by the Bradford method using bovine serum albumin as standard $\left(1 \mathrm{mg}\right.$ of protein corresponds to $\mathrm{ca}$. $2.5 \mathrm{x} 10^{6}$ J774A.1 and TS/A cells ${ }^{24}$ ).

\section{Evaluation of detection threshold for Dy-labeled cells.}

Dilution experiments on Dy-labeled cells were performed in order to evaluate the lowest number of labeled cells able to generate a detectable CEST contrast. Dy-labelled J774A.1 cells were counted by using a Burker chamber. Then they were mixed with different amount of unlabelled cells (from $100 \%$ to $12 \%$ ) and introduced into glass capillaries, centrifuged at $1000 \mathrm{rpm}$. MRI CEST images of these mixed pellets were acquired. 


\section{Assessment of cells toxicity of DyHPDO3A complexes.}

The viability of cells labeled with DyHPDO3A by macropinocytosis or hypotonic swelling was assessed. Both TS/A and J774A.1 cells were counted by Trypan Blue exclusion assay, before and after the labeling with 100mM DyHPDO3A. As control, the similar number of cells was incubated (macropinocytosis or hypotonic swelling) in presence of a corresponding volume of PBS. The experiments were repeated three times.

In vivo visualization of Dy-labelled cells.

The in vivo experiments were carried out on ten week-old female Balb/c mice (Charles River Laboratories, Calco, Italy). They were treated in accordance with the University Ethical Committee and European guidelines. $0.1 \mathrm{ml}$ of a suspension containing $3 \times 10^{6}$ Dy-labeled TS/A cells (labelled with $100 \mathrm{mM}$ Dy-HPDO3A by macropinocytosis) were subcutaneously inoculated in the flank of the mice. The MR image was acquired $24 \mathrm{~h}$ after the injection in order to allow the re-absorption of liquids. For the MR images acquisition, mice were anesthetized by intramuscular injection of tiletamine/zolazepam (Zoletil 100; Virbac, Milan, Italy) 20 mg/kg plus xylazine (Rompun; Bayer, Milan, Italy) $5 \mathrm{mg} / \mathrm{kg}$. The in vivo experiments were carried out in triplicate.

\section{MRI experiments and data analysis}

Z-spectra were acquired at 7T on a Bruker Avance 300 spectrometer equipped with a microimaging probe (inner coil $10 \mathrm{~mm}$ or $30 \mathrm{~mm}$ for in vitro and in vivo experiments, respectively). A frequency offset range of \pm 25 ppm was investigated. A typical RARE spin-echo sequence (RARE factor 8) with an echo time of $3 \mathrm{~ms}$ and a TR value of $5 \mathrm{~s}$ was used. An acquisition matrix of $64 \times 64$ with a square FOV of $10 \mathrm{~mm}$ or $35 \mathrm{~mm}$ was used for in vitro or in vivo experiment, respectively. The whole sequence was preceded by a saturation scheme consisting of a continuous rectangular block pulse wave with a $B_{1}$ of $3 \mu \mathrm{T}$ and duration of $2 \mathrm{~s}$ or $1.5 \mathrm{~s}$ for in vitro or in vivo experiments, respectively. The Z-spectra were interpolated by smoothing splines to identify, firstly, the correct position of the bulk water and, then, the correct ST\% value over the range of frequency offset investigated. Custom-made software, compiled in the Matlab platform (Mathworks Inc., Natick, MA), was used ${ }^{25,26}$. A quantitative CEST study is typically conducted by an asymmetric analysis approach, i.e. two image acquisitions with irradiation at the CEST offset ( $\Delta \omega)$ and the opposite offset $(-\Delta \omega)$ related to water signal are collected and computed to determine the percentage CEST ratio called Saturation Transfer (ST): 


$$
S T \%=\left(1-\frac{M S(\Delta \omega)}{M S(-\Delta \omega)}\right) \times 100
$$

in which $\operatorname{MS}(\Delta \omega)$ and MS $(-\Delta \omega)$ are the MR signal intensity of the bulk water protons upon applying a saturation pulse at $\Delta \omega$ and - $\Delta \omega$, respectively.

\section{$\underline{\text { Results }}$}

\section{Effects of Shift Reagent loading inside cells}

Ln-HPDO3A complexes ( $\mathrm{Ln}=\mathrm{Gd}$, Eu, Dy, Tm, Fig.S1) have been loaded inside cells, in the endosomes (via macropinocytosis) or in the cytosol (via hypotonic swelling), respectively. Then, pellets have been analyzed by MRI. Fig.1A and $B$ report, respectively, $T_{2 w^{-}}$and $T_{1 w^{-}}$weighted MR Images of a phantom containing cellular pellets of J774A.1 cells labeled with the four complexes as localized in the endosomes or in the cytosol, respectively. The signal intensity of the capillaries containing cells loaded with SRs in the endosomes appears lower, because of the stronger $\mathrm{T}_{2}$ * effect (Fig.1A). In $T_{1, w}$ image (Fig.1B), signal intensity is higher for cells containing Gd-HPDO3A (capillary 2 and 6). Table1 reports $\mathrm{T}_{1}$ and $\mathrm{T}_{2}$ of all samples. Fig.1C-J reports Z- and ST\%-spectra of all the samples. For unlabelled cells, a signal at $2.5 \mathrm{ppm}$ is present, due to endogenous exchangeable protons. In all the other samples, which contain cells labeled with paramagnetic STs, a more shifted CEST signal is detectable. The magnitude of the shift of the intracellular water resonance $\left(\delta^{\ln }\right)$ strictly depends on the used Ln-HPDO3A and on its localization, varying from a minimum of 2.9 ppm (for Eu-HPDO3A labelled cells) to a maximum of 8 ppm (for Dy-HPDO3Alabelled cells).

Fig.2A shows the comparison among the ST profiles of cells labeled by using Eu- Tm- or DyHPDO3A by pinocytosis. Among the three tested shift reagents, Dy-HPDO3A showed the higher shifting ability (ca.8 ppm). The observed $\delta^{\ln }$ values are related to the second power of the effective magnetic moment of the Lanthanide ion ( $\mu_{\text {eff }} \mathrm{Dy}=10.6 ; \mu_{\text {eff }} \mathrm{Tm}=7.6$, Eu= 3.5, Fig.2B). ${ }^{19,20,22,29}$

\section{Assessment of in vitro performance of Cell-CEST}

Insights into the sensitivity of such agents have been gained by acquiring CEST MR images of mixtures of Dy-labelled cells and unlabelled cells. Fig.3 shows the $T_{2 w}$ images (A) and the ST calculated maps (B) of mixtures with different percentages of labeled cells (from ca. $10 \%$ to 75\%). As shown in Fig.3C, by decreasing the amount of Dy-labelled cells there is a decrease of ST\% but a 
clear contrast is still evident even in the sample with the lowest amount of labeled cells (where only ca. $10 \%$ of cells were labeled). Therefore the detection threshold appears to be lower than $10 \%$ of labeled cells.

As a proof of concept for the detection of two sets of cells in the same image, murine breast cancer (TS/A) cells have been labelled with Tm-HPDO3A by hypotonic swelling and J774A.1 cells have been labelled with Dy-HPDO3A by pinocytosis. Fig.4 shows the ST maps (depicted in false color, Fig.4B,C,D) superimposed on the $\mathrm{T}_{2 \mathrm{~W}}$ image (Fig.4A) of a phantom consisting of glass capillaries containing mixtures of the two cell populations labelled by pinocytosis and hypotonic swelling. Tm-HPDO3A labeled cells have been detected by irradiating at 3 ppm (Fig.4B) while DyHPDO3A labeled cells have been detected by irradiating at $8 \mathrm{ppm}$ (Fig.4C). The merge of the two ST maps allows localizing the different cells type in the same MR image (fig.4 D). ST\% spectra of the samples have been reported in Fig.S2.

\section{$\underline{\delta^{\ln } \text { as reporter of the cellular proliferation rate }}$}

The herein reported approach has been used for assessing in vitro and in vivo cell proliferation rate. Dy-labelled cells have been collected at different times after the labeling (from $t=0$ to $t=4$ days) and CEST-MR images have been acquired to measure the $\delta^{\ln }$ value. In Fig.5 results obtained with J774A.1 and TS/A cells have been reported (left column for TS/A cells and right column for J774A.1 cells). ST\% spectra have been reported in Fig. S3. There is an overall reduction of the observed chemical shift upon time, with an exponential decay behavior that reflects the different growth constant (K) of the two cell types (Fig.5A and B). The exponential decay is faster for TS/A than for J774A.1 cells ( $K=-0.043$ vs -0.027 , respectively). Cell proliferation can also be monitored by following the $\mathrm{R}_{2}{ }^{*}$ changes. In fact, the herein used Ln-complexes, and especially Dy-HPDO3A, display also a strong $\mathrm{T}_{2}{ }^{*}$ effect. Since $\mathrm{R}_{2}{ }^{*}$ of cells depends on the relative intracellular amount of Dysprosium (as well as from its location), the measurement of $\mathrm{R}_{2}{ }^{*}$ reports on the cellular concentration/distribution of the paramagnetic probe. The $\mathrm{R}_{2}{ }^{*}$ effect reflects the CEST response, i.e. $\mathrm{R}_{2}{ }^{*}$ exponentially decreases upon time after the labeling step, with a time constant $(\mathrm{k})$ similar to the one measured from the changes of $\delta^{\ln }$ (Fig.5C and D).

Next, it has been shown that the $\delta^{\ln }$ and $\mathrm{R}_{2}{ }^{*}$ changes are inversely related to the amount of cells at the different time points (Fig.5 E and F). The proliferation rate displays a mono-exponential 
behavior with a growth constant that is equal and opposite to the one obtained from the changes in the $\delta^{\mathrm{ln}}$ and $\mathrm{R}_{2}{ }^{*}$ (Fig.5). In the Table1 the obtained K-values are reported.

\section{$\underline{\text { In vivo tests }}$}

Finally, Dy-HPDO3A-labelled TSA cells have been subcutaneously injected in the right flank of a mouse to evaluate if it possible to visualize the labelled cells in vivo (yellow arrow, Fig. 6A). As control unlabelled TS/A cells have been injected in the left flank of the same mouse (white arrow, Fig. 6A). As shown in Fig.6B it is possible to detect in vivo the Cell-CEST contrast at $4.8 \mathrm{ppm}$ from water signal (image acquired $24 \mathrm{~h}$ after the injection). The chemical shift of the ST peak has been monitored for the few days in order to evaluate the in vivo cell proliferation rate during the development of the tumor. ST\% spectra have been reported in Fig. S4. In vivo, the estimated K value for the proliferation rate of TS/A cells is -0.031 .

\section{Discussion}

\section{Effects of Shift Reagent loading inside cells}

Ln-HPDO3A complexes ( $\mathrm{Ln}=\mathrm{Gd}$, Eu, Dy, Tm, Fig.S1) have been chosen as paramagnetic shift reagents because i) they are chemically analogues of the clinically approved Gadoteridol, ii) they are small, hydrophilic and neutral molecules that appear well tolerated also at high intracellular concentration both if localized inside cell cytoplasm or in the endosomes ${ }^{27}$. Pinocytosis and hypotonic swelling have been used as labeling procedures to reach similar overall intracellular Lanthanide payloads.

The loading by macropinocytosis leads the internalization of the Ln-complexes in the intracellular vesicles, since they are entrapped by membrane invagination followed by intracellular trafficking of lipidic vesicles (endosomes). Conversely, the application of hypotonic swelling leads the loading of the Ln-complexes in the cytoplasm, since they cross the cellular membrane through transient pores opening in the membrane and moved by gradients of concentration. Therefore, the hypotonic swelling route is expected to yield a more homogeneous distribution of the SR in the cytoplasm in respect to the one obtained by macropinocytosis ${ }^{23,24}$. About $1.5 \times 10^{10} \operatorname{Ln}(\mathrm{III})$-HPDO3A molecules per cell have been entrapped, that corresponds to an intracellular concentration of $\operatorname{Ln}(I I I)-H P D O 3 A$ of $c a .10 \mathrm{mM}$. 
The effect of intracellular payload of high amounts of paramagnetic Ln-HPDO3A complexes inside cells (entrapped either by macropinocytosis and hypotonic swelling) was extensively investigated in the past years and no toxicity has noted even at high intracellular concentrations of the entrapped probe. ${ }^{16,18,23,24,30}$ Herein, cell-viability experiments on cells labelled with Dy-HPDO3A complex (loaded by macropinocytosis and hypotonic swelling) have been carried out and they confirmed the overall absence of toxicity. A slight effect on cells viability is due to the hypotonic swelling procedure; in fact it occurs either upon DyHPDO3A labeling or when the procedure is carried out in the absence of the metal complexes by using buffer as control (Fig. S5).

Cells have been imaged by MRI immediately after their detachment. The image acquisition is generally 15-20 minutes long. During this time cells are perfectly intact, with preserved membranes. Cells have been imaged by MRI immediately after their detachment. The image acquisition took ca 15-20 minutes. During this time cells appear intact, with preserved membranes. After this time cells appear intact, with well preserved membranes at the microcopy control. We cannot exclude processes of cells death begin during this time. What we can exclude is the release of the metal complexes from cells because i) the ICP-MS analysis did not show any metal-containing species in the extracellular fluid and ii) the release of SRs from cells would have led to the decrease, and eventually the disappearance, of the CEST effect.

The presence of the paramagnetic SRs largely affect the $T_{2}{ }^{*}$ of the cell pellets; as expected, when the $S R$ is localized in the endosomes, its effect on the $T_{2}{ }^{*}$ is larger with respect to when it is distributed in the cytoplasm (Fig.1A and B). Furthermore, $\mathrm{T}_{2}{ }^{*}$ shortening is higher in the specimens labelled with Dysprosium and smaller in the ones labelled with Europium.

The presence of Gd-HPDO3A largely affects also the water proton $T_{1}$, especially when the probe is distributed in the cytoplasm. As previously reported, when high amounts of Gd- complexes are loaded inside the endosomes, a "quenching" of $\mathrm{T}_{1}$ may occur ${ }^{28}$ (Fig. 1B and Table S1). CEST experiments have been performed at room temperature by applying a RF pulse of $3 \mu \mathrm{T}$ on a range of $\pm 25 \mathrm{ppm}$ with respect to the bulk water signal. Unlabeled cells show only the characteristic right-hand asymmetry of the Z-spectrum and the corresponding ST peak centered at ca. $2.5 \mathrm{ppm}$ downfield the water resonance. This asymmetry can be accounted for in terms of the pool of exchanging protons of soluble peptides and proteins, naturally occurring in cells' cytoplasm (Fig.1C-J black lines) $^{14,19,20 .}$.

Conversely, in the case of cells labeled with the paramagnetic SRs, the Z-spectrum and the ST values are markedly different from the control (Fig.1C-J). The magnitude of the shift of the 
intracellular water resonance $\left(\delta^{\ln }\right)$ strictly depends on the used Ln-HPDO3A and on its localization. For example, when Dy-HPDO3A has been loaded inside cells endosomes, the ST peak is centered at $8 \mathrm{ppm}$ from water, with a ST\% of $c a .20 \%$. The increased bandwidth is due to the overall decrease of $\mathrm{T}_{2}{ }^{*}$ as consequence of the large paramagnetic loading but the marked asymmetry of the Z-absorption and the net shift and enhancement of the ST peak is a clear evidence that the cellular container, loaded with the paramagnetic Dy-HPDO3A, acts as CEST agent.

This effect, as previously reported for Red Blood Cells loaded with Dy-HPDO3A, cannot be simply accounted in term of a dipolar shift. ${ }^{19,20,29}$ In fact, the estimated dipolar term for an intracellular concentration of Dy-HPDO3A of $c a .10 \mathrm{mM}$ corresponds to ca. $-0.2 \mathrm{ppm}$ (negative since Dysprosium Bleaney's constant is negative and the dipolar shift is equal to $-19 \mathrm{ppm} / \mathrm{M})^{19,20,29}$.

The Z-spectra from cells loaded by hypotonic swelling yielded narrower linewidth with respect to those loaded by pinocytosis. This behavior is due to differences in the $T_{2}{ }^{*}$ values, being much shorter for those cells with an inhomogeneous distribution of the paramagnetic complexes that causes larger magnetic susceptibility effects (BMS). When BMS effects are larger, the induced shifts are greater but also $\mathrm{T}_{2}{ }^{*}$ decreases; as a consequence the Z-spectra may became noisier because of the fast decay of the signal. It is worth noting that Gd labeled cells (Fig.1 G,H) showed a higher saturation transfer effect when the complexes are into the endosomes than when they are in the cytosol. The reason of this difference is ascribed to the fact that when the Gd complexes are compartmentalized into the endosomes the $T_{1}$ of the bulk water is less affected. ${ }^{26,29}$. Saturation transfer efficiency is directly proportional to the longitudinal relaxation time, thus the shorter is the $T_{1}$ the lower is the saturation transfer. For these reasons, cells containing $\mathrm{Gd}$ HPDO3A in the endosomal compartment give rise to a higher ST effect (ST\% ca.18\% vs. ca.5\% observed upon labeling with hypotonic swelling). When cells are labelled with other Ln-complexes (e.g. Eu-, Tm-, Dy-HPDO3A) no significant difference in the extent of observed ST\% has been detected whatsoever hypotonic swelling or pinocytosis labeling procedure has been applied(Fig.1C,D,E,F,IJ).

The comparison among the ST profiles of cells labeled by using Eu- Tm- or Dy-HPDO3A by pinocytosis has been reported in Fig.2A. $\delta^{\ln }$ values are related to the second power of the effective magnetic moment of the Lanthanide ion; hence Dy-HPDO3A, which has the highest effective magnetic moment ( $\mu^{\text {eff }}$ provides the highest $\delta^{\text {ln }}$

When the SRs are dispersed in the cytoplasmatic space (higher homogeneity) the shift is significantly smaller. 


\section{Assessment of in vitro performance of Cell-CEST}

More insights into the sensitivity may be gained by looking at specimens in which Dy-HPDO3A has been confined inside the cytosol (Fig.3). Dy-HPDO3A labeled cells have been diluted with unlabelled cells and ST\% effect has been measured. It has been chosen to mix labeled cells with unlabelled ones because it more closely mimics the in vivo condition of an ideal experiment of tracking of ex vivo labeled cells. In fact, when Ln-labeled cells are administrated in vivo, it is expected that they are naturally embedded in the tissue, becoming only a small part of the entire tissue which is composed mainly by unlabelled cells. ${ }^{30}$

For this measurement, different amounts of Dy-labelled cells have been mixed with unlabelled. $T_{2 w}$ images (Fig.3A) and the ST calculated maps (Fig.3B) shows that lower the amount of labelled cells, lower the ST\% signal. A detectable and clear contrast is still evident even in the sample with the lowest amount of labeled cells (where only ca. $10 \%$ of cells were labeled) (Fig.3C). Therefore the detection threshold appears to be lower than $10 \%$ of labeled cells.

Cell as CEST agents can be used also for multicolor CEST MRI. As reported in Fig.4, Tm-labelled TS/A cells and Dy-labelled J774A.1 cells can be simultaneously visualized in a false color MR image. The first set of cells is characterized by the intracellular shifted water signal with a maximum ST\% at ca. $3.0 \mathrm{ppm}$ whereas the second one at about $6.8 \mathrm{ppm}$, with an overlap of the two ST curves. Therefore, the offset of irradiation for both cells populations has been properly chosen in order to minimize the overlapping between the respective ST spectra, i.e. at $3 \mathrm{ppm}$ for Tm-labelled TS/A cells and at 8 ppm for Dy-labelled J774A.1 cells.

\section{$\underline{\delta^{\ln } \text { as reporter of the cellular proliferation rate }}$}

Finally, the above described approach to generate Cell-CEST systems has been applied as tool to evaluate the in vitro and/or in vivo cell proliferation rate. During the time after the labeling, cells proliferates. Upon successive mitosis, the paramagnetic content distributed in the cytoplasm of the labelled cells will progressively decrease in the daughter-cells. This process affects either the value of the intracellular water chemical shift $\left(\delta^{\mathrm{in}}\right)$ or the shape of ST\% distribution function. (The latter effect is the result of the interplay between the amounts of intracellular water and the membrane permeability to the water molecules.)

$\delta^{\text {in }}$ appears the more amenable reporter of the underlying proliferation process. $\delta^{\text {in }}$ values have been extracted from the maxima of the ST\% measured at the different time points. In the days after the labeling, it changes with a decrease that has an exponential behavior. This exponential 
decay reflects the different growth constant $(K)$ of the two cell types, indicating that TS/A cells proliferate faster than J774A.1 cells (Fig.5A and B). In fact, faster the proliferation, quicker the reduction of intracellular payload of SRs with consequent decreasing of $\delta^{\text {in }}$. The decrease of the intracellular SRs content also affects $R_{2}$ which decreases the days after the labeling (Fig.5C and D). K-values obtained by the exponential fitting of $\delta^{\text {in }}$ and $R_{2} v s$. time curves are in line with those ones obtained upon proliferation assays. (Fig.5 E, F).

\section{$\underline{\text { In vivo tests }}$}

Cells labelled with paramagnetic SRs can be detected when administrated in vivo, for instance in the flank of mice (Fig.6, yellow arrow). The monitoring of $\delta^{\ln }$ value can report about in vivo proliferation index of cells. At $\mathrm{t}=24 \mathrm{~h}$ post cells administration, $\delta^{\ln }$ is equal to $4.8 \mathrm{ppm}$. in the days after, this values decreases, because of the cells proliferation and consequent reduction of intracellular concentration of SR molecules. From the fitting of the curve, it is possible to notice a slower proliferation rate of TS/A in vivo respect to what happens when the same cells are cultivated in vitro ( $\mathrm{K}=-0.031$ in vivo vs. $\mathrm{K}=-0.04$ in vitro).

It is to be noted that it was not possible to measure $\delta^{\ln }$ value at $\mathrm{t}=0$ ( i.e. immediately after the administration of cells) because of the presence of liquid in the region of cells administration.

\section{Conclusions}

In this work new insights into the effects of paramagnetic Shift Reagents on intracellular water have been reported. It has been shown that Red Blood Cells are not the only cell type able to act as CEST agents upon loading with SRs. The Cell-CEST contrast displays a very high sensitivity since the large pool of intracellular water protons is used as CEST pool of exchanging spins. It has been found that the chemical shift depends on the amount of paramagnetic complexes entrapped inside the cells, on the metal complex that has been used (different for $\mu_{\text {eff }}$ ) and on the intracellular localization of the probe (free in the cytosol or entrapped into endosomes). In particular if the Lanthanide(III) ion is represented by $\mathrm{Gd}^{3+}$, the endosomal compartmentalization is advantageous since the shortening of $T_{1}$ is detrimental for the generation of the saturation Transfer and the "quenching " of the relaxation enhancement, occurring when the probe is in the endosomes, becomes advantageous. Regarding the other Lanthanides, the ensodomal compartmentalization causes an increase of the BMS contribution and a decrease of $\mathrm{T}_{2}{ }^{*}$ thus yielding broadening of the ST peaks that may make necessary to increase the acquisition times. 
The herein reported labeling system is feasible for in vivo applications and two representative examples have been reported. The first one is in the field of multiple cells visualization that may open the way to applications in new cell tracking studies by MRI. The second application has dealt with the evaluation of the cellular proliferation rate. Both in vitro and in vivo the chemical shift of the intracellular water signal changes because of the naturally occurring cell division that causes the dilution of the SRs that are inside the cells.

Although this work was conceived to assess the practicability of Cell-CEST concept beyond the previously reported Erythro-CEST systems, the obtained results provide some insights into the understanding of the observed CEST effect. Although far from the asymmetrical shape of RBCs, the shape of the cells types considered in this work are certainly not spherical. Therefore, in principle, the distribution of paramagnetic species in an asymmetrical compartment leads to a marked BMS contribution that affects both $\delta^{\mathrm{In}}$ and $\mathrm{T}_{2} *$. However, the differences observed in $\delta^{\mathrm{ln}}$ and $\mathrm{T}_{2} *$ where the SR is distributed in the cytoplasm or it is encapsulated in the endosomes suggest that the BMS term receives a contribution from an asymmetric distribution of the paramagnetic complexes in the cellular volume. Likely, the paramagnetic systems move in the cells to seek for optimal interactions with the magnetic flux lines. The high paramagnetic load of the endosomes may cause an even more prompt response to find a proper location for these vesicles. Overall we may expect an anysotropic distribution of paramagnetics in the cells that contribute to generate a marked BMS contribution. The movement of magnetic particles- loaded endosomes in the presence of a static magnetic field was early reported by F. Gazeau et al. in studies aimed at assessing the origin of the BMS effect generated by iron oxide superparamagnetic particles ${ }^{31,32}$.

\section{Acknowledgments:}

We gratefully acknowledge Bracco Imaging for providing ProHance. Support by European H2020 H2020-PHC-2015 IDentiFY project is acknowledged. G.F. and E.D.G were supported by FIRC-AIRC (Fondazione Italiana per la Ricerca sul Cancro AIRC) fellowships.

\section{$\underline{\text { References }}$}

1. Edmundson M, Thanh NT, Song B. Nanoparticles based stem cell tracking in regenerative medicine. Theranostics 2013; 3: 573-82.

2. Kanno H. World Regenerative therapy for neuronal diseases with transplantation of somatic stem cells. J. Stem Cells 2013; 5: 163-171.

3. Weissleder R, Mahmood U. Molecular Imaging. Radiol 2011; 219: 316-33. 
4. Himmelreich U, Dresselears T. Cell labeling and tracking for experimental models using magnetic resonance imaging. Methods 2009; 48:112-24.

5. Srinivas M, Boehm-Sturm P, Figdor CG, de Vries IJ, Hoehn M. Labeling cells for in vivo tracking using (19)F MRI. Biomaterials 2012; 33: 8830-40.

6. Geraldes CF, Laurent S. Classification and basic properties of contrast agents for magnetic resonance imaging. Contrast Media Mol Imaging 2009; 4: 1-23.

7. Sherry AD, Caravan P, Lenkinski RE. Primers on gadolinium chemistry. J. Magn. Reson. Imaging 2009; 30: 1240-8.

8. Caravan P. Strategies for increasing the sensitivity of gadolinium based MRI contrast agents. Chem. Soc. Rev. 2006; 35: 512-23.

9. Modo M, Hoehn M, Bulte JW. Cellular MR imaging. Mol Imaging 2005; 4: 143-64.

10. Frank JA, Anderson SA, Kalsih H, Jordan EK, Lewis BK, Yocum GT, Arbab AS. Methods for magnetically labeling stem and other cells for detection by in vivo magnetic resonance imaging. Cytotherapy 2004; 6: 621-5.

11. Bulte JW, Kraitchman DL. Iron oxide MR contrast agents for molecular and cellular imaging.NMR Biomed. 2004; 17: 484-99.

12. Ward KM, Aletras AH, Balaban RS. A new class of contrast agents for MRI based on proton chemical exchange dependent saturation transfer (CEST). J. Magn. Reson. 2000; 143: 79-87.

13. Wu Y, Evbuomwan M, Melendez M, Opina A, Sherry AD. Advantages of macromolecular to nanosized chemical-exchange saturation transfer agents for MRI applications. Future Med Chem. 2010; 2: 351-66.

14. van Zijl PC, Yadav NN. Chemical exchange saturation transfer (CEST): what is in a name and what isn't? Magn. Reson. Med. 2011; 65: 927-48.

15. McMahon MT, Gilad AA, DeLiso MA, Berman SM, Bulte JW, van Zijl PC. New "multicolor" polypeptide diamagnetic chemical exchange saturation transfer (DIACEST) contrast agents for MRI. Magn. Reson. Med. 2008; 60: 803-12.

16. Ferrauto G, Delli Castelli D, Terreno E, Aime S. In vivo MRI visualization of different cell populations labeled with PARACEST agents. Magn. Reson. Med. 2013; 69: 1703-11.

17. Pumphrey A, Yang Z, Ye S, Powell DK, Thalman S, Watt DS, Abdel-Latif A, Unrine J, Thompson K, Fornwalt B, Ferrauto G, Vandsburger M. Advanced cardiac chemical exchange saturation transfer (cardioCEST) MRI for in vivo cell tracking and metabolic imaging. NMR Biomed. 2016; 29: 74-83.

18. Nicholls F, Ling W, Ferrauto G, Aime S, Modo M. Simultaneous MR imaging for tissue engineering in a rat model of stroke. Sci. Rep. 2015; 5: 14597.

19. Ferrauto G, Delli Castelli D, Di Gregorio E, Terreno E, Aime S. LipoCEST and cellCEST imaging agents: opportunities and challenges. Wiley Interdiscip. Rev. Nanomed. Nanobiotechnol. 2016; 8: 602-18.

20. Ferrauto G, Delli Castelli D, Di Gregorio E, Langereis S, Burdinski D, Gruell H, Terreno E, Aime S. Lanthanide-loaded erythrocytes as highly sensitive chemical exchange saturation transfer MRI contrast agents. J. Am. Chem. Soc. 2014; 15: 638-41.

21. Ferrauto G, Di Gregorio E, Baroni S, Aime S. Frequency-encoded MRI-CEST agents based on paramagnetic liposomes/RBC aggregates. Nano Lett. 2014; 14: 6857-62.

22. Corsi DM, Iglesias CP, van Bekkum H, Peters JA. Determination of paramagnetic lanthanide(III) concentrations from bulk magnetic susceptibility shifts in NMR spectra Magn. Res. Chem., 2001; 11: 723-726.

23. Gianolio E, Stefanìa R, Di Gregorio E, Aime S. MRI paramgnetic probes for cellular labeling. Eur. J. Inorg. Chem. 2012; 12: 1934-1944.

24. Di Gregorio E, Ferrauto G, Gianolio E, Aime S. Gd loading by hypotonic swelling: an efficient and safe route for cellular labeling. CMMI. 2013; 8: 475-86. 
25. Stancanello J, Terreno E, Delli Castelli D, Cabella C, Uggeri F, Aime S. Development and validation of a smoothing-splines-based correction method for improving the analysis of CEST-MR images. CMMI. 2008; 4: 136-149.

26. Terreno E, Stancanello J, Longo D, Delli Castelli D, Milone L, Maarten BK, Uggeri F, Aime S. Methods for an improved detection of the MRI-CEST effect. CMMI, 2009; 4: 237-247.

27. Di Gregorio E, Gianolio E, Stefània R, Barutello G, Digilio G, Aime S. On the fate of MRI Gd-based contrast agents in cells. Evidence for extensive degradation of linear complexes upon endosomal internalization. Anal. Chem. 2013; 85: 5627-5631.

28. Strijkers G, Hak S, Kok MB, Springer CS Jr, Nikolay K. Three-compartment T1 relaxation model for intracellular paramagnetic contrast agents. Magn. Res. Med. 2009; 61: 1049-58.

29. Peters JA, Huskens J, Raber D, Lanthanide induced shifts and relaxation rate enhancements. J. Prog. Nucl. Magn. Reson. Spectrosc. 1996, 28, 283-350.

30. Delli Castelli D, Ferrauto G, Di Gregorio E, Terreno E, Aime S. Sensitive MRI detection of internalized T1 contrast agents using magnetization transfer contrast. NMR Biomed. 2015; 28: 1663-70.

31. Wilhelm C, Gazeau F. Universal cell labelling with anionic magnetic nanoparticles. Biomaterials 2008; 29: 3161-74.

32. Wilhelm C, Lavialle F, Pechoux C, Tatischeff I, Gazeau F. Intracellular trafficking of magnetic nanoparticles to design multifunctional biovesicles. Small 2008; 5: 577-582.

Table

\begin{tabular}{cccc}
\hline & K values from $\delta^{\ln }$ & K values from $R_{2}$ & K values from cells' count \\
\hline TS/A cells & $-\mathbf{0 . 0 4 3} \pm \mathbf{0 . 0 0 3}$ & $-\mathbf{0 . 0 3 7 \pm 0 . 0 0 3}$ & $\mathbf{0 . 0 4 0 \pm 0 . 0 0 2}$ \\
J774A.1 cells & $\mathbf{- 0 . 0 2 7 \pm 0 . 0 0 2}$ & $\mathbf{- 0 . 0 2 7 \pm 0 . 0 0 1}$ & $\mathbf{0 . 0 2 2 \pm 0 . 0 0 3}$ \\
\hline
\end{tabular}

Table 1. $K$ values obtained for TSA and J774A.1 cells.

\section{Figures and tables Captions}

Fig.1: A) T2w and B) T1w Image of a phantom containing: 1 unlabelled cells; 2-5 cells labeled by pinocytosis with Gd-, Eu-, Tm- or Dy-HPDO3A; 6-9 labeled by hypotonic swelling with Gd-, Eu-, Tm- or Dy-HPDO3A. C,E,G,I) Z-spectra and D,F,H,J) ST-spectra of Dy-, Eu-, Gd-, and Tm-HPDO3Alabelled cells. Dotted lines correspond to cells labeled by hypotonic swelling, continues lines to cells labeled by pinocytosis.

Fig.2 A) Comparison among ST profiles of cells labelled by pinocytosis with Dy- (blue), Eu- (red) or Tm-(green) HPDO3A at the same intracellular concentration. B) Relationship between the induced chemical shift and the effective magnetic moment of the Lanthanide. The number of LnHPDO3A/Cell is maintained constant for all the cellular samples to $c a .1 .5 \times 10^{10} \mathrm{Ln} /$ cell.

Fig.3: A) $T_{2}$ weighted image and B) ST map of a phantom containing 1) unlabelled cells, 2) $100 \%$ Dy labeled cells, 3) $75 \%$ Dy labeled cells and $25 \%$ unlabelled cells, 4) $50 \%$ Dy labeled cells and $50 \%$ unlabelled cells, 5) $25 \%$ Dy labeled cells and $75 \%$ unlabelled cells, 6) $12.5 \%$ Dy labeled cells and $87.5 \%$ unlabelled cells. C) ST\% vs. concentration of labelled cells.

Fig.4: A) T2w Image, B) STmap@3ppm, C) STmap@10 ppm ,D) merge of B) and C), of a phantom containing: 1) unlabeled cells, 2)TS/A cells labeled with Tm-HPDO3A in the cytosol, 3) J774A.1 cells labeled with Dy-HPDO3A in the endosomes and 4) pellet containing $50 \%$ of cells 1 and $50 \%$ of cells 2 
Fig.5: Evaluation of proliferation rates for TS/A (A, C, E) and J774A.1 (B, D, F) cells. (A, B) Decreasing of chemical shift during cell division; (C, D) Decreasing of $R_{2}$ during cell division; ( $\left.E, F\right)$ Cell proliferation.

Fig.6: A) In vivo visualization of Dy-labelled TSA cells ( yellow arrow) $24 \mathrm{~h}$ post injection by irradiating at $4.8 \mathrm{ppm}$. Unlabelled TSA cells have been injected as control (white arrow) ; B) Decreasing of chemical shift inside tumor region during the days post injection of Dy-labelled TSA cells.

Table 1. K values obtained for TSA and J774A.1 cells.

Fig.S1. Chemical structure of Ln-HPDO3A ( $\mathrm{Ln}=\mathrm{Eu}, \mathrm{Gd}$, Dy or $\mathrm{Tm})$ complexes

Fig.S2. ST\% spectra of specimens reported in Fig.4 i.e. 1) unlabelled cells as control (grey line), 2) TS/A cells labelled with TmHPDO3Aby hypotonic swelling (green line), 3) J774A.1 cells labelled with DyHPDO3A by macropinocytosis (red line) and 4) a mixture of Tm-labelled-TS/A and Dy-labelled-J774A.1 cells (yellow line).

Fig.S3. ST\% spectra of (A) of Dy-labelled-TS/A cells and (B) Dy-labelled-J774A.1 cells at different times after the labeling (T=0, 24h, 48h, 72h and 96h)

Fig.S4. ST\% spectra in vivo administrated Dy-labelled-TS/A at different times after the labeling and administration (T=0, 24h, 48h, 72h and 96h)

Fig.S5. (A) J774A.1 cells viability upon loading of DyHPDO3A 100mM by macropinocytosis or hypotonic swelling; (B) TS/A cells viability upon loading of DyHPDO3A 100mM by macropinocytosis or hypotonic swelling. Hypotonic swelling and macropinocytosis with buffer solution have been used as control.

Table.S1. $\mathrm{T}_{1}$ and $\mathrm{T}_{2}$ measure $\left(7 \mathrm{~T}, 2^{\circ} \mathrm{C}\right)$ of J774A.1 cells labelled with Ln-complexes by pinocytosis or hypotonic swelling. 